\title{
INFLUENCE OF DIFFERENT SINTERING PROTOCOLS ON TRANSLUCENCY AND FRACTURE RESISTANCE OF MONOLITHIC ZIRCONIA CROWNS
}

\author{
Rasha Sayed Asaad* and Mostafa Elsayed Aboushahba**
}

\begin{abstract}
Purpose : The current study investigated the effect of different sintering protocols (long, speed and high- speed cycles) on translucency and fracture resistance of monolithic zirconia crowns.

Materials and Methods: 30 monolithic zirconia crowns were CAD/CAM fabricated from translucent BruxZir zirconia blanks. The crowns were divided into three groups $(\mathrm{n}=10$ each) according to the sintering protocols (temperature \& speed); Group (I): samples sintered by long sintering cycle $\left(1510^{\circ} \mathrm{C}, 120 \mathrm{~min}\right.$ holding time and 8 hours total cycle time), Group (II): speed sintering $\left(1540^{\circ} \mathrm{C}, 25\right.$ min holding time and 2 hours total cycle time) and Group (III): high-speed sintering $\left(1580^{\circ} \mathrm{C}, 10 \mathrm{~min}\right.$ holding time representing the total firing cycle). Translucency parameter (TP) and Contrast Ratio (CR) were measured using a spectrophotometer, then compressive load was applied till fracture in a universal testing machine. One representative disc sample $(10 \mathrm{~mm}$ diameter x $1 \mathrm{~mm}$ thickness) was fabricated and sintered according to the parameters of each group and analyzed by scanning electron microscope. Collected data were statistically analyzed.
\end{abstract}

Results: Sintering speed had a statistically significant effect on (TP), (CR) and fracture resistance means. Long cycle showed the statistically significantly highest (TP) and lowest (CR) mean values. The high-speed cycle recorded the statistically significantly highest fracture resistance mean values.

Conclusion: Shortening the sintering cycle significantly decreased translucency and increased fracture resistance of monolithic zirconia crowns. Regarding Translucency Perception Threshold, speed cycle can be recommended for sintering of BruxZir monolithic zirconia crowns.

KEYWORDS: Monolithic zirconia; Translucent zirconia; Translucency; Fracture resistance; Sintering protocols.

* Oral and Maxillofacial Prosthodontic Department, Faculty of Dentistry, King Abdulaziz University, Jeddah, Kingdom of Saudi Arabia.

** Lecturer Fixed Prosthodontics Faculty of Oral and Dental Medicine. Future University in Egypt, Cairo, Egypt 


\section{INTRODUCTION}

Zirconia has been recently employed as a highly attractive ceramic material due to its superior mechanical properties that cannot be found in any other ceramic system owed to the transformation toughening mechanism in addition to its better natural appearance in comparison to metal-ceramics ${ }^{(1,2)}$. Zirconia has some outstanding properties such as less plaque accumulation, excellent biological compatibility, low corrosion and minimal thermal conductivity ${ }^{(3,4)}$. Therefore, zirconia can be used in fabrication of many fixed prostheses as crowns, fixed partial dentures, implant fixtures and implant abutments ${ }^{(5)}$. However, low translucency and hydrothermal instability are considered as challenges being faced when using zirconia-based restorations ${ }^{(6)}$. Related to their high opacity resulting from the decreased amount of light transmission and much scattering through the restoration, zirconia cores are always veneered with porcelain to imitate the appearance of natural teeth. The most common clinical failures occurred in these veneered restorations is the cracking or chipping of the veneering porcelain as setting a long-lasting mechanical or chemical bond between zirconia and veneering porcelain was confirmed to be difficult. In addition to the low fracture strength of these veneered restorations ${ }^{(7,8)}$.

To overcome the problems encountered in zirconia with veneering porcelain, full-contour monolithic zirconia restorations have been introduced for their more easier production as no additional veneer layer is needed, resulting in decreased fabrication time and cost. Many researches have encouraged the incorporation of monolithic zirconia in clinical cases with excessive masticatory forces, pathological attrition and of inadequate interocclusal distance related to its ability to resist high loads with only $0.5 \mathrm{~mm}$ occlusal thickness ${ }^{(8-10)}$. Additionally, monolithic zirconia have become greatly popular as it is associated with decreased amount of tooth prepa- ration and thickness of restorative material when compared to veneered zirconia restoration as well as minimal wear of opposing enamel in contact to polished monolithic zirconia ${ }^{(7,11,12)}$. Moreover, monolithic zirconia crowns revealed higher fracture strength than veneered zirconia crowns ${ }^{(8,13-15)}$. On the other hand, other investigators showed similar strength in monolithic and veneered zirconia ${ }^{(16)}$. Owing to these studies, it can be suggested that monolithic zirconia can be used as an alternative to veneered zirconia.

One of the most important challenges for monolithic zirconia restorations is to obtain acceptable esthetic outcome as they are a mono-layered restorations. According to translucency, zirconia is classified into; opaque and translucent. The opaque zirconia is characterized by greater strength, so it is usually indicated to be used as posterior restorations. The translucent zirconia promotes more natural esthetic appearance by providing better translucency upon sintering to be used as both posterior and anterior restorations. In-order to increase the translucency for monolithic zirconia, some attempts that were concerned with the modification in fabrication techniques, coloring techniques, and sintering process have been applied to change some of zirconia properties ${ }^{(\mathbf{1 4})}$.

The sintering process is considered as one of the utmost important stage in the fabrication of zirconia restorations in which it might be modified in order to optimize the properties of zirconia. Although, $\mathrm{CAD} / \mathrm{CAM}$ techniques have reduced the clinical operating times significantly, the sintering process still takes long durations. Alterations in sintering parameters have shown a strong influence in dental researches particularly following the presentation of short sintering cycles by manufacturers as rapid sintering can be accomplished in minutes resulting in the fabrication of zirconia restorations in a single visit and subsequently, increase its use clinically ${ }^{(17,18)}$. 
Some researchers found that increasing the sintering temperature and/or durations have resulted in larger tetragonal zirconia grain sizes which are declared to increase translucency ${ }^{(18-20)}$. But, the large grain size can cause spontaneous tetragonal to monoclinic transformation (T-M), which consequently decrease the material stability resulting in a gradual decrease in strength ${ }^{(\mathbf{2 1})}$. Accordingly, lower temperature and/or time reduce the grain size, at which (T-M) transformation does not occur, and the strength is at the highest level in small grains ${ }^{(22)}$.

The influence of variations in sintering temperature and time on the grain size, translucency and flexural strength of zirconia have been studied by many researchers ${ }^{(7,10,18,20)}$; still the consequence of these alterations on fracture resistance of zirconia crowns remains questionable. So the objective of this study was to explore the effect of changing sintering protocols (long, speed and high-speed cycles) on translucency and fracture resistance of monolithic translucent zirconia crowns. The null hypothesis was that different sintering protocols would not affect translucency and fracture resistance of zirconia crowns.

\section{MATERIALS AND METHODS}

\section{Study Design:}

In this in-vitro study, 30 monolithic zirconia crowns were CAD/CAM fabricated from translucent zirconia blanks (BruxZir ${ }^{\mathrm{TM}}$ shaded, Glidewell, Frankfurt, Germany). A maxillary right second premolar tooth in a typodont model (Frasaco $\mathrm{GmbH}$, Tettnang, Germany) was prepared according to the standard tooth preparation procedures with deep chamfer finish line $(1 \mathrm{~mm})$, occlusal reduction $(1.5 \mathrm{~mm})$, axial reduction $(1 \mathrm{~mm})$, and $\left(6^{\circ}\right)$ angle of convergence ${ }^{(8)}$. Then, the prepared tooth was scanned and the crowns were designed, milled and finally sintered according to the protocol of each group. The CAD/CAM fabricated monolithic zirconia crowns were divided into three groups
( $\mathrm{n}=10 \mathrm{each}$ ) according to the sintering protocols (temperature and speed); Group (I): long sintering (sintering at $1510^{\circ} \mathrm{C}$ for $120 \mathrm{~min}$ holding time and 8 hours total cycle time), Group (II): speed sintering (sintering at $1540^{\circ} \mathrm{C}$ for 25 min holding time and 2 hours total cycle time) and Group (III): high-speed sintering (sintering at $1580^{\circ} \mathrm{C}$ for 10 min holding time representing the total firing cycle).(TP) and (CR) were measured utilizing a spectrophotometer. The typodont prepared tooth was duplicated into (30) epoxy resin dies. All the crowns were cemented on their corresponding epoxy dies and then subjected to compressive load till fracture in a universal testing machine. One representative disc sample (10mm diameter x $1 \mathrm{~mm}$ thickness) was fabricated and sintered according to the sintering protocol of each group and analyzed by scanning electron microscope.

\section{Fabrication of monolithic Zirconia crowns}

\section{Digital scanning (Optical impression)}

The prepared second premolar tooth was digitally scanned using a three dimensional (3D) dental scanner (Identica hybrid blue scanner, MEDIT T 300, Seoul, Korea). The Medit system is an open system that can export 3D data as a (Standard Tessellation Language) STL file. The resulting scan was converted to STL format and sent directly to the lab.

\section{Designing of the crowns}

The designing process for the full-contour monolithic crowns was performed by the aid of the CAD software (Exocad Dental CAD,v.2016,GmbH, Darmstadt, Germany). The minimum material thickness was set to $1 \mathrm{~mm}$ and the spacer thickness was set to $40 \mu \mathrm{m}$. Data were transported to the computer connected milling machine to start milling full-contour monolithic zirconia crowns following the manufacturer instructions. 


\section{Milling of the crowns:}

Translucent zirconia blanks ( BruxZir ${ }^{\mathrm{TM}}$ shaded blanks, Glidewell, Frankfurt, Germany) of shade A2 were used in this study which is composed of (Zirconium Oxide $\mathrm{ZrO}_{2}<89 \%$, Yttrium Oxide $\mathrm{Y}_{2} \mathrm{O}_{3}$ $>6 \%$, Hafnium Oxide $\mathrm{HfO}_{2}>4 \%$, Aluminum Oxide $\left.\mathrm{Al}_{2} \mathrm{O}_{3}>1 \%\right)$.

Zirconia blanks were inserted into a 5-axis milling machine(vhf CAM 5-S1, camfacture AG, Germany). The crowns were milled with a $20 \%$ enlargement as specified by the manufacturer to compensate for the sintering shrinkage. After milling ,a specific BruxZir finishing bur was used to separate the crowns from the blank. Then all crowns were cleaned ultrasonically in distilled water for $10 \mathrm{~min}$ utes. The crowns were then placed into the drying unit (Robocam Thermostar, Aachen, Germany) for 5 minutes at a temperature of $80^{\circ} \mathrm{C}$.

\section{Sintering of the crowns:}

After finishing of the milling process ,the crowns were inserted in a high temperature sintering furnace (in fire HTC speed, Sirona Dental Systems)according to the following sintering temperatures and speeds:

- Long cycle (Group I): Crowns were located over the sintering beads in the sintering tray which was loaded into the furnace at room temperature, then the temperature was gradually increased till reaching the sintering temperature $\left(1510^{\circ} \mathrm{C}\right)$ which was held for 120 minutes, after that the crowns were cooled down to room temperature. The total cycle time was 8 hours.

- Speed cycle (Group II): Crowns were sintered at $\left(1540^{\circ} \mathrm{C}\right)$ sintering temperature that was held for 25 minutes. The total cycle time was 2 hours.

- High-speed cycle (Group III): The empty furnace was first preheated till $\left(1580^{\circ} \mathrm{C}\right)$ then the crowns were placed in the heated furnace and kept for 10 minutes sintering time. After that, the furnace was opened and the crowns were taken out for bench cooling. The total cycle time was 10 minutes.

Then, all sintered crowns were glazed to attain a smooth surface following the manufacturer instructions.

\section{Fabrication of Zirconia dises}

\section{Designing of the disc}

Designing of a zirconia disc (12 $\mathrm{mm}$ diameter $\mathrm{x}$ $5 \mathrm{~mm}$ thickness) was done by using a CAD software (Exocad Dental CAD, v.2016, GmbH, Darmstadt, Germany). The disc shape was designed in the form of 2D model.

\section{Milling\& sintering of the discs}

A disc sample (12mm diameter x $5 \mathrm{~mm}$ thickness) was milled following the manufacturer instructions. Then, the disc sample was cut into disc slices (12mm diameter $\times 1.2 \mathrm{~mm}$ thickness) of presintered BruxZir using water cooled saw (ISOMET 4000 , Buehler, Lakebluff. USA) that is $20 \%$ larger than the final size to recompense for shrinkage that took place through sintering process, so that final dimensions of discs would be $(10 \mathrm{~mm}$ diameter $\mathrm{x}$ $1 \mathrm{~mm}$ ) thickness after sintering. Disc samples were sintered similar to crown samples of each group, then the thickness of each disc was inspected using a digital caliper.

\section{Testing Procedures}

\section{Translucency Measurements}

\section{Translucency Parameter (TP)}

Translucency was tested using Vita Easy shade spectrophotometer (Vita, Ivoclar, Vivadent AG, Schann, Liechtenstein). Each crown was seated on the prepared tooth, tightly secured in its place using a specially designed holding device. The $\mathrm{L}^{*}, \mathrm{a}^{*}$ and $b^{*}$ was measured for each crown by placing the probe tip for Vita Easy shade on the central part of 
the buccal surface of the crown ${ }^{(23)}$. To guarantee the consistency of measurements, probe tip was placed at the same place on each crown. All color measurements were repeated 3 times by the same examiner for each crown. The crowns were placed against white and black backgrounds. Then the $\mathrm{L}^{*}, \mathrm{a}^{*}, \mathrm{~b}^{*}$ mean values were recorded. Before each measurement the device was calibrated for standardization.

The (TP) values were calculated following this equation:

$$
\mathbf{T P}=\left[\left(\mathbf{L}_{\mathbf{b}}^{*}-\mathbf{L}_{\mathrm{w}} *\right)^{2}+\left(\mathbf{a}_{\mathrm{b}} *-\mathbf{a}_{\mathrm{w}}^{*}\right)^{2}+\left(\mathbf{b}_{\mathbf{b}} *-\mathbf{b}_{\mathrm{w}}^{*}\right)^{2}\right]^{1 / 2}
$$

As, "b" and "w" are color coordinates against black and white background, respectively. In all calculations, "0" was considered as totally opaque and "100" as totally transparent. As the (TP) value increases, a material will be more translucent ${ }^{(24)}$.

\section{Contrast Ratio $(\mathrm{CR})$ :}

The contrast ratio (CR) for each crown was calculated according to the following equations:

The $L^{*}$ values were used to calculate the spectral reflectance $(\mathrm{Y})$ as follows:

$\mathbf{Y}=\left[\left(\mathbf{L}^{*}+\mathbf{1 6}\right) / \mathbf{1 1 6}\right]^{3} \mathbf{x 1 0 0}$. Where, the values recorded on white $(\mathrm{Yw})$ and black $(\mathrm{Yb})$ backgrounds were used to calculate the (CR) as in this equation:

$\mathbf{C R}=\mathbf{Y b} / \mathbf{Y w}$. $\mathbf{C R}$ values range from 0 (transparent material) to 1 (completely opaque material).

\section{Fracture resistance testing:}

The prepared tooth was duplicated into thirty epoxy resin dies (Kemapoxy165, Egypt). The monolithic zirconia crowns were cemented on corresponding epoxy dies with self-adhesive resin cement (E-Cem DC, Bisico, Bielefelder GmbH \&Co. KG) under constant axial load of $5 \mathrm{~kg}$ using a specially constructed cementing device, then resin cement was light cured from all aspects of each crown, followed by storing in $100 \%$ humidity at $37^{\circ} \mathrm{C}$ for 24 hours. Then all zirconia crowns were individually mounted on a computer controlled testing machine
(Model 3345; Instron Industrial Products, Norwood, MA, USA) with a load cell of 5 kN. Figure1 Compressive load was applied occlusally at cross-head speed of $1 \mathrm{~mm} / \mathrm{min}$. Failure loads were determined and their values were recorded in Newton(N).

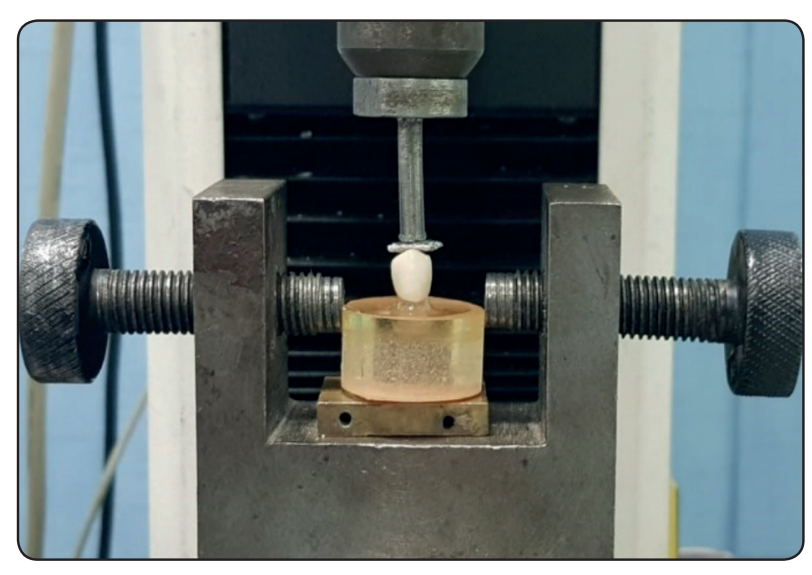

Fig. (1): A universal testing machine

\section{Environmental Scanning Electron Microscopic (SEM) Analysis:}

After sintering of the disc samples, they were ultrasonically cleaned for $10 \mathrm{~min}$ using distilled water and dried with oil free compressed air. Environmental Scanning Electron Microscope (QuantaFEG-ESEM250) was used for analysis of the discs (as sintered). The disc samples were examined with accelerating voltage $30 \mathrm{~K} . \mathrm{V}$ and at magnifications of 3000x,30000x \& 60000x.

\section{Statistical Analysis:}

One-way ANOVA test was used to study the influence of sintering speed on mean (TP), (CR) and fracture resistance. Pearson's correlation coefficient was used to study the relation between (TP) and (CR). The significance level was set at $\mathrm{P} \leq 0.05$. Statistical analysis was performed with IBM ${ }^{\circledR}$ SPSS $®$ Statistics Version 20 for Windows. 


\section{RESULTS}

Collected data was explored for normality using Kolmogorov-Smirnov and Shapiro-Wilk tests and showed parametric (normal) distribution.

\section{Translucency Results}

\section{Translucency Parameter (TP)}

One-way ANOVA revealed that the sintering speed had a statistically significant effect on (TP) mean. The long cycle had the statistically significantly highest mean (TP). There was a statistically significant difference between long, speed and highspeed cycles; where high-speed had the statistically significantly lowest (TP) mean values. Table 1

\section{Contrast Ratio (CR)}

One-way ANOVA revealed that the sintering speed had a statistically significant effect on (CR) mean.

The high-speed cycle had the statistically significantly highest mean (CR). There was a statistically significant difference between long, speed and highspeed cycles; as long cycle had the statistically significantly lowest (CR) mean values. Table 2

TABLE (1): Descriptive statistics and results of oneway ANOVA for (TP) values of different groups

\begin{tabular}{|c|c|c|}
\hline \multirow{2}{*}{ Sintering cycle } & \multicolumn{2}{|c|}{ Translucency } \\
\cline { 2 - 3 } & Mean & SD \\
\hline Long cycle & $13.263^{\mathrm{a}}$ & 0.616 \\
\hline Speed cycle & $11.603^{\mathrm{b}}$ & 0.300 \\
\hline High-speed cycle & $10.040^{\mathrm{c}}$ & 0.217 \\
\hline p-value & \multicolumn{2}{|c|}{$<\mathbf{0 . 0 0 1 *}$} \\
\hline
\end{tabular}

Different letters indicate statistically significance difference. *; significant at $p \leq 0.05$
TABLE (2): Descriptive statistics and results of oneway ANOVA for (CR) values of different groups

\begin{tabular}{|c|c|c|}
\hline \multirow{2}{*}{ Sintering cycle } & \multicolumn{2}{|c|}{ Contrast ratio } \\
\cline { 2 - 3 } & Mean & SD \\
\hline Long cycle & $0.663^{\mathrm{c}}$ & 0.012 \\
\hline Speed cycle & $0.707^{\mathrm{b}}$ & 0.025 \\
\hline High-speed cycle & $0.770^{\mathrm{a}}$ & 0.010 \\
\hline p-value & \multicolumn{2}{|c|}{$\mathbf{0 . 0 0 1}^{*}$} \\
\hline
\end{tabular}

Different letters indicate statistically significance difference. ${ }^{*}$; significant at $p \leq 0.05$

\section{Correlation between TP and CR}

Pearson correlation revealed that there was a statistically significant negative relation between (TP) and $(\mathrm{CR})$ with coefficient of correlation. $(\mathrm{r}=-0.877$, $\mathrm{P}$-value $=0.002$ )

\section{Fracture resistance results}

One-way ANOVA revealed that the sintering speed had a statistically significant effect on fracture resistance mean. There was a statistically significant difference between the three sintering cycles; where the high-speed cycle had the statistically significantly highest mean values and the long cycle had statistically significantly lowest mean values.

Table 3

TABLE (3): Descriptive statistics and results of oneway ANOVA for fracture resistance values of different groups

\begin{tabular}{|c|c|c|}
\hline \multirow{2}{*}{ Sintering cycle } & \multicolumn{2}{|c|}{ Fracture resistance } \\
\cline { 2 - 3 } & Mean & SD \\
\hline Long cycle & $1407.040^{\mathrm{c}}$ & 13.231 \\
\hline Speed cycle & $1676.227^{\mathrm{b}}$ & 21.510 \\
\hline High-speed cycle & $1988.897^{\mathrm{a}}$ & 10.729 \\
\hline $\boldsymbol{p}$-value & \multicolumn{3}{|c|}{$<\mathbf{0 . 0 0 1}{ }^{*}$} \\
\hline
\end{tabular}

Different letters indicate statistically significance difference. *; significant at $p \leq 0.05$ 


\section{Scanning Electron Microscopic results:}

(SEM) images of representative disc samples of each sintering cycle are illustrated in Figure 2 to show the microstructure of zirconia. The analysis of the images revealed obvious difference in zirconia grain size as an outcome of different sintering speeds. Sample of long sintering cycle showed large and irregularly shaped grains, size ranged from 400$800 \mathrm{~nm}$ with the predominance of large grains and increased porosity than the other groups Figure 2A. While sample of speed cycle showed slightly smaller grains than those of long cycle that varied from 200-500 $\mathrm{nm}$ with a more homogeneous grain size distribution, irregular angular-shaped grains and less porosity than long cycle Figure 2B. However, sample of high-speed cycle showed the smallest grain size that ranged from 100-300 nm with dominant areas of small grains, of relatively regular rounded grain shape in addition to increased grain boundaries with more densely packed and least porous structure. Figure 2C
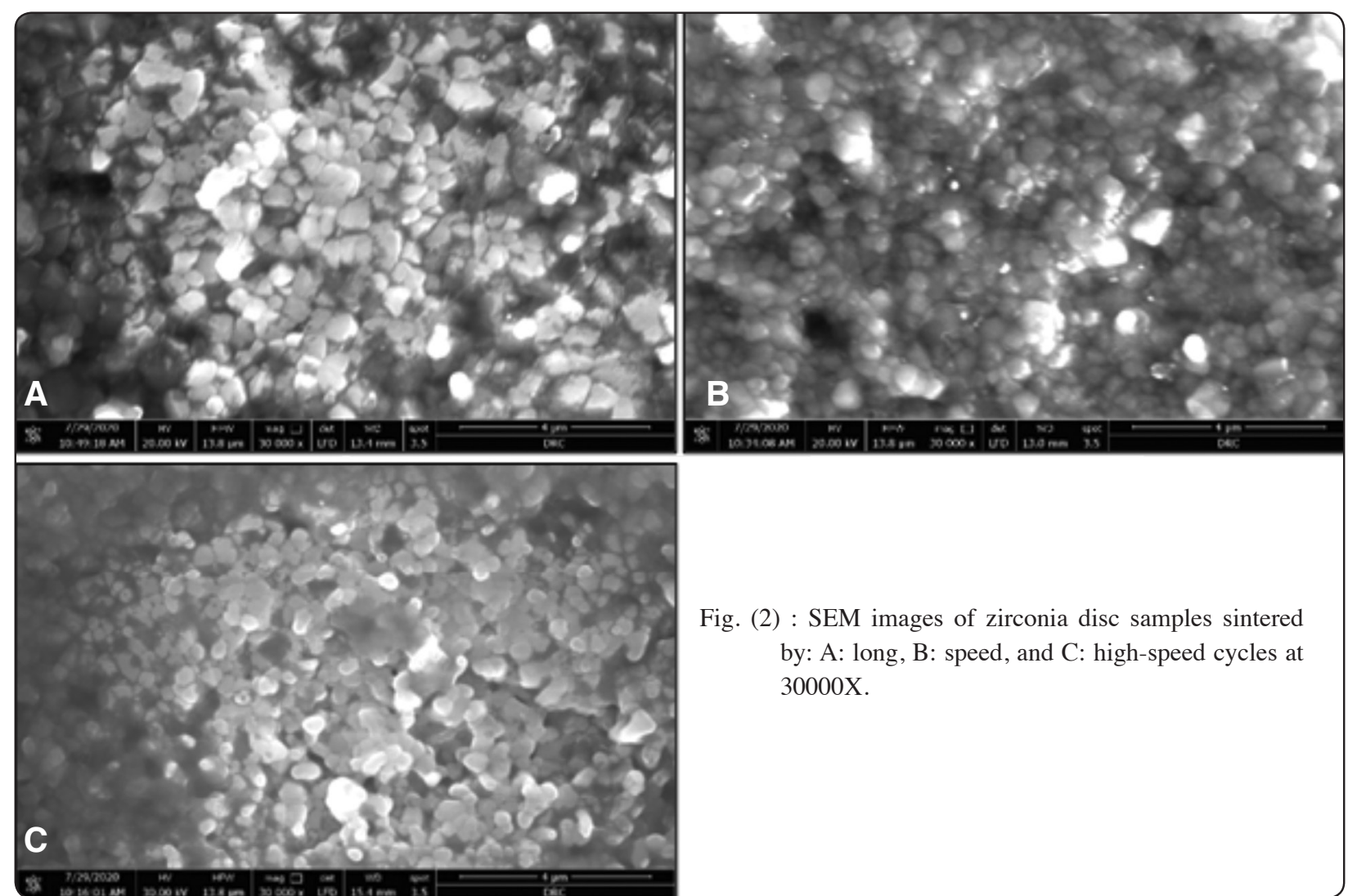

Fig. (2) : SEM images of zirconia disc samples sintered by: A: long, B: speed, and C: high-speed cycles at 30000X. 


\section{DISCUSSION}

Monolithic translucent zirconia has been emerging as a promising option due to its favorable optical and mechanical properties ${ }^{(13,25)}$. In this study, "BruxZir" crowns were selected as they had proven high fracture strength and minimal wear to the opposing dentition than other monolithic translucent zirconia crowns ${ }^{(8)}$.

This study was carried out in response to the request of reducing cost and time to produce a single visit chair side CAD/CAM prostheses, as it promote shortening of the sintering cycle duration following the previous researches that investigated how altering the sintering parameters affect both optical and mechanical properties of monolithic zirconia and subsequently its clinical serviceability as fixed prostheses $^{(7,18)}$.

Esthetic ceramic prostheses should match both color and translucency of natural teeth. So translucency is the most determining factor that influences esthetic properties of ceramic restorations. An esthetic material's translucency and opacity could be evaluated by different methods as: absolute translucency (direct light transmittance), and relative translucency measured either by (TP) or (CR). In this study, both (TP) and (CT) were chosen to evaluate the translucency of monolithic zirconia crowns. As the restoration is considered absolutely opaque if the (TP) is equal to zero and absolutely transparent if the (TP) is equal to "100"(24). Whereas, (CR) value is " 0 " for a transparent material and " 1 "for a completely opaque material ${ }^{(26,27)}$. In this study, there was an agreement with Della Bona et al. ${ }^{(28)}$ who established a statistical correlation between (TP) and $(\mathrm{CR})$ results $(\mathrm{r}=-0.877, \mathrm{P}$-value $=0.002)$.

The majority of researches used flat disc samples of standardized thickness to evaluate translucency of ceramic materials; but in this current study, monolithic full-anatomical zirconia crowns were selected instead of disc samples for measuring translucency in order to be more clinically relevant.
Which was in accordance with some researches that measured color and translucency through the buccal surface of various types of ceramic crowns ${ }^{(13,29-31)}$. Moreover, using the spectrophotometer to analyze color has many benefits, the most important one is handling color as a numerical variable which allow accurate comparison of different parameters without the interference of the effect of the surrounding light source or reflections ${ }^{(32)}$.

Resistance to fracture is an important determinant of a material's mechanical properties. Most of the researchers had chosen flexural strength in evaluating the influence of altering sintering protocols on mechanical properties of zirconia flat samples ${ }^{(32-34)}$. For this reason, this study had evaluated the influence of changing sintering parameters on fracture resistance of cemented translucent monolithic full anatomical zirconia crowns; for both reliability and suitability of those restorations on clinical basis. Additionally, zirconia crowns were cemented with resin cements owing to studies that reported higher fracture strength in ceramic restorations cemented with adhesive resin cements ${ }^{(35)}$.

Many researches informed that the alterations in the sintering parameters of zirconia affect its grain size and microstructure and thereby affect (TP) and (CR), therefore (SEM) analysis was carried out in this study to find the relation between them ${ }^{(7,17,22,32)}$.

In this research, the use of different sintering protocols statistically significantly affected both the translucency and fracture resistance. These results led to rejection of the null hypothesis which stated that different sintering protocols would not affect translucency and fracture resistance of zirconia crowns.

Concerning the effect of sintering speeds on translucency, shortening of the sintering cycle resulted in a decrease in translucency and increase of opacity. The long sintering cycle registered the highest (TP) and lowest (CR) in comparison to the other two cycles. This result might be related to the 
effect of sintering parameters on the grain size as the longer the holding time (120 min.) and sintering duration ( 8 hours) the larger the grain size, as it was proved that zirconia grain size is considered the most crucial determinant of its translucency and also it might be due to fewer grain boundaries leading to increased light transmission and subsequently increasing translucency ${ }^{(18,19,27,33)}$. These findings were supported by SEM images of the long cycle's disc sample that showed the largest grain size with decreased number of grain boundaries among the tested groups Figure 2A. This was in accordance with some investigators who showed that both higher sintering temperature and longer holding time increased the grain growth resulting in large grain size in long cycle $>$ speed cycle $>$ high-speed cycle $^{(17,18,27,32-34)}$. These translucency results were in agreement with many studies which concluded that increasing the holding time during sintering process enhances translucency of monolithic zirconia restorations ${ }^{(32,33,35)}$. In addition to other studies which revealed that the translucency was increased in parallel with the increasing of grain size ${ }^{(20,36)}$. Also, the results were aligned with Ebied et al. ${ }^{(33)}$ who concluded that sintering BruxZir zirconia using longer holding time would result in decreased contrast ratio and better translucency.

The crowns of speed sintering cycle recorded lower translucency than that of long cycle which was due to the presence of smaller grains and more grain boundaries as demonstrated in the SEM images. Figure 2B The short holding time (25 min.) and sintering duration ( 2 hours) might be the cause of decreasing the grain size and increasing grain boundaries in comparison to the long cycle samples $^{(37)}$. However, the lowest (TP) and highest (CR) was found in the high-speed cycle which might be due to the existence of the smallest grain size and more increased number of grain boundaries than the other two cycles as shown in the SEM image Figure 2C . This was in agreement with Denry I \& Kelly $\mathbf{J R}^{(38)}$ who declared that the amount of grain bound- aries influence translucency depending on the grain size, as the decreased grain size always resulted in increased amount of grain boundaries and subsequently, a decrease in translucency is recorded.

These findings were not coinciding with those of Kaizer et al. ${ }^{(29)}$ who compared the translucency of monolithic translucent zirconia crowns using three sintering cycles. They recorded translucency of low values for all groups with slightly better performance of high-speed group. This contradiction might be attributed to the difference in sintering speeds for the long and speed cycles as the total sintering time was 4 hours \& 60 minutes, respectively. Additionally, for imaging the microstructure through SEM, they used crown samples with flat surface that was thermally etched at $1150{ }^{\circ} \mathrm{C}$ which is completely different from the current study as we used disc samples as sintered for microstructure examination.

Also, our results were against those of Coskun ME \& Sari $\mathbf{F}^{(39)}$ who found better translucency of samples in high-speed cycle. This opposition might be due to the use of multilayered monolithic zirconia where no obvious grain size difference was detected among the groups according to the SEM images. Also, Kim et al. ${ }^{(17)}$ concluded that shortening the sintering time could result in more translucent zirconia restorations, which might be owed to different zirconia brands and sintering conditions between both studies.

Regarding the results of fracture resistance, a significant difference was detected between all tested groups as high-speed cycle had the highest mean among the three groups while the long cycle had the lowest. Based on the relationship between the microstructure and mechanical properties of zirconia, previous studies found that the transformation toughening effect, strength and toughness were dependent on the grain size ${ }^{(7,21)}$. Also, it was found that strength is at its highest level in small grains as the fracture size increases in relation to the grain size ${ }^{(22)}$.

The increase in fracture resistance of the highspeed cycle $\left(1580^{\circ} \mathrm{C}, 10 \mathrm{~min}\right)$ might be related to the 
decrease in grain size in addition to more regularly rounded shaped grains showing a homogenous, less porous microstructure as revealed in SEM images Figure 2C. As this increased temperature $\left(1580^{\circ} \mathrm{C}\right)$ might improve the structure of zirconia crystals with reduced pores, flaws and defects. These findings might be attributed to that shortening the holding time during sintering allowed relatively less capability of phase shifting from tetragonal to monoclinic phase which could result in increasing the mechanical properties. This was in agreement with Casllas et al. ${ }^{(40)}$ who reported that decreasing the grain sizes resulted in enhanced strength.

The results were also consistent with Khaledi et al. ${ }^{(41)}$ who found that the shorter the sintering time, the higher is the compressive strength. The results were additionally convenient with Tekeli $\mathbf{S} \& \mathbf{E r}$ dogan $\mathbf{M}^{(42)}$ who reported that the sintering time influenced the density and mechanical properties of zirconia as they concluded that high sintering temperature and prolonged sintering time enlarged the grain size resulting in a material with decreased mechanical properties. Moreover, these findings were in agreement with Ersoy et al. ${ }^{(7)}$ who proved that the high-speed sintering cycle revealed the highest strength among the three sintering cycles using the same parameters as the current study. Also, In line with these findings, some researchers ${ }^{(21,37)}$ reported that as the grain size increases, zirconia become less stable and more subjected to spontaneous tetragonal-monoclinic phase transformations, which might result in gradual reduction of strength. Contrary to this study, Stawarczyk et al. ${ }^{(20)} \&$ Hjerppe et al. ${ }^{(34)}$ showed that short sintering time for zirconia decreased the grain size; but, the results didn't affect the mechanical properties significantly. Also, our findings were against Juntavee $\mathbf{N} \&$ Attashu $\mathbf{S}{ }^{\left({ }^{(43)}\right.}$ who found that either increasing sintering temperature or holding time allows enhancing strength of monolithic zirconia and this contradiction might be due to the difference in sintering temperatures and holding times between both studies. Furthermore, Ebied et al. ${ }^{(33)}$ found that strength of BruxZir shaded zirconia was not affected by alterations in sintering parameters as they used different sintering temperatures and holding times.

According to the Translucency Perception Threshold (TPT), Liu et al. ${ }^{(44)}$ declared that the (TPT) for people was (0.07), above which it can be estimated as clinically perceivable by $50 \%$ of non-professional persons and below which is clinically undetectable to people. It was evaluated in the current study through calculating $(\triangle \mathrm{CR})$ between any two groups. It was found that $(\triangle \mathrm{CR})$ between long cycle and speed cycle was (0.044) and it was $(0.063)$ between the speed and the high-speed cycles. So $(\Delta \mathrm{CR})$ values in this study were less than the (TPT $=0.07)$ except between the long cycle and high-speed cycle which was $(0.10)$. This means that both long and speed cycles effect on translucency would not be perceived by people, whereas highspeed cycle effect would be perceivable. $(\Delta \mathrm{CR})$ values were calculated from. Table 2

As the average maximum biting force posteriorly was recorded as $(835 \mathrm{~N})$ for females and $(999 \mathrm{~N}$ )for males. So, with regard to the fracture resistance results in this study; the zirconia crowns of the three sintering cycles accomplished a higher fracture load than the biting forces in the posterior region ${ }^{(45)}$.

Although , this study used anatomical monolithic translucent zirconia crowns for evaluating translucency and fracture resistance in order to be more clinically relevant. But, still have some limitations; First, one brand of zirconia ceramic was evaluated. Second, fracture of samples was carried out under static load which is less representative of clinical masticatory forces than the dynamic fatigue tests. Further in vivo studies are required in the future to evaluate effect of changing sintering parameters on optical and mechanical properties of monolithic zirconia restorations. 


\section{CONCLUSION}

1. Shortening the sintering cycle significantly decreased translucency and increased fracture resistance of monolithic zirconia crowns.

2. Regarding Translucency Perception Threshold, speed cycle can be recommended for sintering of BruxZir monolithic zirconia crowns.

\section{REFERENCES}

1. Okuda Y, Noda M, Kono H, Miyamoto M, Sato H, Ban S. Radio-opacity of core materials for all ceramic restorations. Dent Mater. 2010;29(1):35-40.

2. Noda M, Okuda Y, Tsuruki J, Minesaki Y, Takenouchi Y, Ban S. Surface damages of zirconia by Nd:YAG dental laser irradiation. Dent Mater. 2010;29(5):536-41.

3. Conrad HJ, Seong WJ, Pesun IJ. Current ceramic materials and systems with clinical recommendations: a systematic review. J Prosthet Dent. 2007;98(5):389-404.

4. Guazzato M, Albakry M, Ringer SP, Swain MV. Strength, fracture toughness and microstructure of a selection of allceramic materials. Part ii. Zirconia-based dental ceramics. Dent Mater. 2004;20(5):449-56.

5. Ban S. Reliability and properties of core materials for allceramic dental restorations. Japan Dent Sci Rev. 2008; 44(1):3-21.

6. Denry I , Holloway JA. Ceramics for Dental Applications: A Review. Materials 2010; 3(1):351-68.

7. Ersoy NM, Aydoğdu HM, Değirmenci BÜ, Çökük N, Sevimay $M$. The effects of sintering temperature and duration on the flexural strength and grain size of zirconia. Acta Biomater Odontol Scand. 2015;1(2-4):43-50.

8. Sarikaya I, Hayran Y. Effects of dynamic aging on the wear and fracture strength of monolithic zirconia restorations. BMC Oral Health 2018;18(1):146.

9. Freire Y, Gonzalo E, Lopez-Suarez C, Suarez MJ. The marginal fit of CAD/CAM monolithic ceramic and metalceramic crowns. J Prosthodont 2017;00:1-6.

10. Zhang H, Kim BN, Morita K, Keijiro Hiraga HY, Sakka Y. Effect of sintering temperature on optical properties and microstructure of translucent zirconia prepared by highpressure spark plasma sintering. Sci Technol Adv Mater. 2011;12(5):055003.
11. Rinke S, Fischer C. Range of indications for translucent zirconia modifications: Clinical and technical aspects. Quintessence Int. 2013; 44(8): 557-66.

12. Rosentritt M, Preis V, Behr M, Hahnel S, Handel G, Kolbeck C. Two body wear of dental porcelain and substructure oxide ceramics. Clin Oral Investig 2012;16: 935-43.

13. Beuer F, Stimmelmayr M, Gueth JF, Edelhoff D, Naumann M. In vitro performance of full-contour zirconia single crowns. Dent Mater. 2012;28(4): 449-56.

14. Zhang Y, Lee JJ, Srikanth R, Lawn BR. Edge chipping and flexural resistance of monolithic ceramics. Dent Mater. 2013;29(12):1201-08.

15. Sun T, Zhou S, Lai R, Liu R, Ma S, Zhou Z .Load-bearing capacity and the recommended thickness of dental monolithic zirconia single crowns. J Mech Behav Biomed Mater.2014;35:93-101.

16. Preis V, Behr M, Hahnel S, Handel G, Rosentritt M. In vitro failure and fracture resistance of veneered and full contour zirconia restorations. J Dent 2012; 40(11): 921-8.

17. Kim MJ, Ahn JS, Kim JH, Kim HY, Kim WC. Effects of the sintering conditions of dental zirconia ceramics on the grain size and translucency. J Adv Prosthodont. 2013;5(2):161-66

18. Stawarczyk B, O“zcan M, Hallmann L, Ender A, Mehl A, Hammerlet $\mathrm{CH}$. The effect of zirconia sintering temperature on flexural strength, grain size, and contrast ratio. Clin Oral Investig. 2013;17:269-74.

19. Pekkan G, Pekkan K, Bayindir BC, O“zcan M, Karasu B. Factors affecting the translucency of monolithic zirconia ceramics: A review from materials science perspective. Dent Mater. 2019;39(1):1-8.

20. Stawarczyk B, Emslander A, Roos M, Sener B, Noack F, Keul C. Zirconia ceramics, their contrast ratio and grain size depending on sintering parameters. Dent Mater J. 2014; 33(5):591-8.

21. Bravo-Leon A, Morikawa Y, Kawahara M, Mayo MJ. Fracture toughness of nanocrystalline tetragonal zirconia with low yttria content. Acta Mater. 2002; 50(18): 4555-62.

22. Eichler J, Rodel J, Eisele U, Hoffman M. Effect of grain size on mechanical properties of submicrometer 3Y-TZP: Fracture strength and hydrothermal degradation. J Am Ceram Soc. 2007; 90(9): 2830-6. 
23. Karaarslan ES, Bulbul M, Yildiz E, Secilmis A, Sari F, Usumez A. Effects of different polishing methods on color stability of resin composites after accelerated aging. Dent Mater J. 2013;32(1):58-67.

24. Kurtulmus-Yilmaz S, Ulusoy M. Comparison of the translucency of shaded zirconia all-ceramic systems. J Adv Prosthodont. 2014;6(5):415-22.

25. Bakitian F, Seweryniak P, Papia E, Larsson C, Vult Von Steyern P. Effect of different semimonolithic designs on fracture resistance and fracture mode of translucent and high translucent zirconia crowns. Clin Cosmet Investig Dent. 2018;10:51-60.

26. Kanchanavasita W, Triwatana P, Suputtamongkol K, Thanapitak A, Chatchaiganan M. Contrast ratio of six zirconia-based dental ceramics. J Prosthodont. 2014 ; 23(6):456-61.

27. Jiang L, Liao Y, Wan Q, Li W. Effects of sintering temperature and particle size on the translucency of zirconium dioxide dental ceramic. J Mater. Sci. Mater Med. 2011;22(11):2429-35.

28. Della Bona A, Nogueira AD, Pecho OE. Optical properties of CAD-CAM ceramic systems. J Dent 2014;42(9): 1202-9.

29. Kaizer MR, Gierthmuehlen PC, Cava SS, Zhang Y. Speed sintering translucent zirconia for chairside one-visit dental restorations: Optical, mechanical, and wear characteristics. Ceram Int. 2017:43(14):10999-11005.

30. Emam ZN , El Sayed SM . Color stability and microstructure changes of two pressable ceramics after repeated firing protocols. EDJ 2020;66(1):453-568.

31. Abd El Aziz MM., Mandour MH, Shetawy RA. Effect of different thickness on fracture strength, marginal accuracy and translucency of monolithic ceramic restorations. ADJfor Girls 2019;6(2):115-22.

32. Sallam HI , Eldwakhly E. Traslucency and biaxial flexural strength of monolithic zirconia as affected by sintering speeds. EDJ 2017;63(2):2083-99.

33. Ebeid K, Wille S, Hamdy A, Salah T, El-Etreby A, Kern M. Effect of changes in sintering parameters on monolithic translucent zirconia. Dent Mater. 2014;30(12):e419-24.

34. Hjerppe J, Vallittu PK, Fröberg K, Lassila LV. Effect of sintering time on biaxial strength of zirconium dioxide. Dent Mater. 2009; 25(2): 166-71.

35. Ahmed KKE. (2016). Effect of changes in sintering parameters on physical properties of monolithic translucent zirconia. (Doctoral Dissertation). Christian-AlbrechtsUniversität zu Kiel. http://macau.uni- kiel.de/receive/ dissertation_diss_00019749.

36. Vichi A, Sedda M, Fabian Fonzar R, Carrabba M, Ferrari $\mathrm{M}$. Comparison of contrast ratio, translucency parameter, and flexural strength of traditional and "augmented translucency” zirconia for CEREC CAD/CAM system. J Esthet. Restor Dent.2016;28(1):32-9.

37. Matsui K, Yoshida H, Ikuhara Y. Isothermal sintering effect on phase separation and grain growth in yttria-stabilized tetragonal zirconia polycrystal. J Am Ceram Soc. 2009;92(2):467-75.

38. Denry I, Kelly JR. Emerging ceramic based materials for dentistry. J Dent. Res. 2014; 93(12):1235-42.

39. Coskun ME, Sari F. Effect of speed sintering on multilayered monolithic zirconia. Cumhuriyet Dent.J 2019; 22(1):31-6

40. Casllas D, Alcala J, Llanes L, Anglada M. Fracture variability and R-curve behavior in yttria-stabilized zirconia ceramics. J Mater. Sci. 2001; 36(12):3011-25.

41. Khaledi AAR, Vojdani M, Farzin M, Pirouzi S. The effect of sintering program on the compressive strength of zirconia copings. J Dent. Shiraz Univ Med Sci.2018;19(3):206-11.

42. Tekeli S, Erdogan M. A quantitative assessment of cavities in $3 \mathrm{~mol} \%$ yttria-stabilized tetragonal zirconia specimens containing various grain size. Ceram Int. 2002; 28(7): 785-9.

43. Juntavee N, Attashu S. Effect of different sintering process on flexural strength of translucency monolithic zirconia. J Clin Exp Dent. 2018;10(8):e821-30.

44. Liu MC, Aquilino SA, Lund PS, Vargas MA, Diaz-Arnold AM, Gratton DG . Human perception of dental porcelain translucency correlated to spectrophotometric measurements. J Prosthodont. 2010;19(3):187-93.

45. Calderon P dos S, Kogawa EM, Lauris JRP, Conti PCR. The influence of gender and bruxism on the human maximum bite force. J Appl. Oral Sci. 2006; 14(6) :448-53. 